\section{Interessenkonflikte und die Glaubwürdigkeit von Leitlinien}

\begin{abstract}
Die Experten, die die Leitlinien anerkannter Organisationen und Fachgesellschaften formulieren, haben oftmals finanzielle Verbindungen mit der Industrie, aber häufig werden diese Konflikte nicht offen gelegt.
\end{abstract}

D ie Autoren werteten Leitlinien zum Thema Hyperlipidämie und Diabetes aus, die von Regierungsorganisationen, nationalen Fachgesellschaften, Ärztegesellschaften oder anderen Nonprofit-Organisationen in den USA und in Kanada zwischen 2000 und 2010 herausgegeben wurden. Ein „Interessenkonflikt“ war definiert als finanzielle Zuwendung für Forschungsarbeiten, Vorträge und Beratung bzw. Aktienbesitz von Firmen, die einschlägige Medikamente vertreiben.

Von 288 Experten, die an 14 Leitlinien beteiligt waren, hatten 150 (52\%) einen Interessenkonflikt. Dieser wurde von zwölf Teilnehmern verschwiegen. Auch jeder zweite Vorsitzende der Leitlinienkommissionen hatte einen Interessenkonflikt.

138 (48\%) bekannten sich zu einem Interessenkonflikt, während 150 dies verneinten oder angaben, keine Gelegenheit zur Erklärung erhalten zu haben. Bei acht von 73 Experten, die einen Interessenkonflikt verneinten, konnten die Autoren solche nachweisen. An den von Regierungsorganisationen geförderten Leitlinien hatten deutlich weniger Mitwirkende einen Interessenkonflikt als bei jenen von NichtRegierungsorganisationen (16 vs. $69 \%$ ).

Neumann J et al. Prevalence of financial conflicts of interest among panel members producing clinical practice guidelines in Canada und United States: cross sectional study. BMJ. 2011;343:d5621. doi: 10.1136/bmj. d5621.

Kommentar von Prof. Heinrich Holzgreve: Diese Analyse dokumentiert die Verbreitung von Interessenkonflikten und weckt Zweifel an der Unabhängigkeit und Objektivität bei der Erstellung von Leitlinien. Kann man dieses Problem lösen? Es wird vorgeschlagen, dass an der Formulierung von Leitlinien nur Experten ohne finanzielle Konflikte mitwirken. Aber dann werden gerade die einflussreichen und erfolgreichen Fachvertreter ausgeschlossen, an denen die Industrie besonders interessiert ist, und der Beraterkreis engt sich erheblich ein. Zitieren wir den Emeritus E. A. M. Gale: „Wir müssen die Realität der Welt, in der wir leben, erkennen. Die akademische und nicht akade-

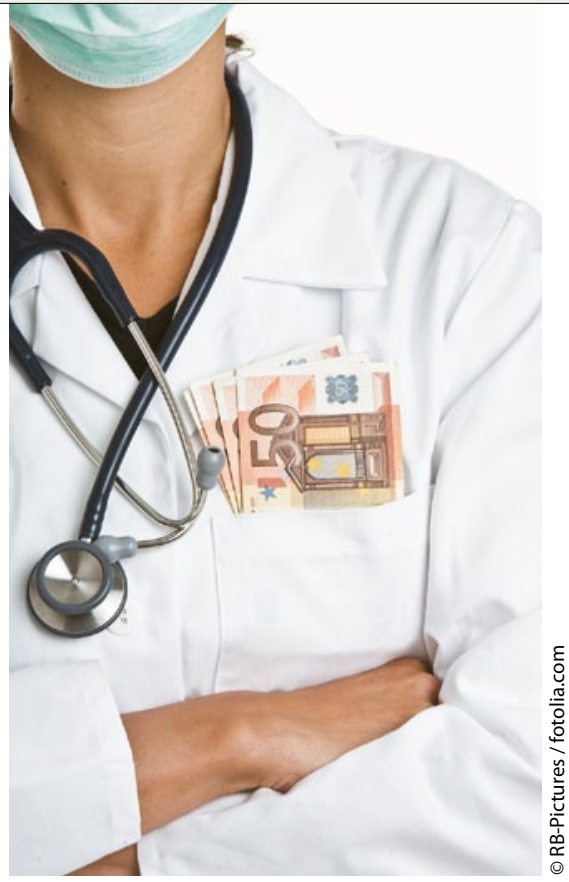

Interessenkonflikte werden nicht immer korrekt gemeldet.

mische Medizin ist durchdrungen von Interessenkonflikten, und viel zu viele profitieren von dieser Situation, um dies öffentlich zu machen. Die Gesetzgebung wird dieses Problem nicht beseitigen, weil das schnelle Geld immer einen Schritt voraus ist."

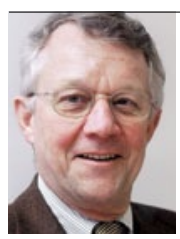

Prof. Dr. med. Heinrich Holzgreve Internist, Kardiologische Praxis, München

\title{
Kopfschmerzgeplagte schlucken zu viele Schmerzmittel
}

\section{Akute Kopfschmerzen lassen sich gut mit den gängigen Schmerzmit- teln behandeln. Schmerzstillende Mittel dürfen jedoch nicht regel- mäßig eingenommen werden.}

n einer Querschnittstudie untersuchten Espen S. Kristofferson und Kollegen, wie in der norwegischen Bevölkerung mit chronischen Kopfschmerzen umgegangen wird.

Von 30.000 befragten Norwegern zwischen 30 und 44 Jahren litten 405 an chronischem Kopfschmerz gemäß ICHD-II (Kopfschmerzen an mehr als 15 Tagen pro Monat). Diese Patienten wurden weiter befragt. 384 litten an chronischen Spannungskopfschmerzen, 15 an Migräne.
$80 \%$ der Befragten hatten aufgrund ihrer Beschwerden den Hausarzt konsultiert, davon wurden $19 \%$ zu einem Neurologen überwiesen und $4 \%$ stationär behandelt. Ein Fünftel $(n=79)$ verzichtete gänzlich auf ärztlichen Rat.

$87 \%$ der Patienten schluckten Schmerzmittel, 9\% sogar täglich. Die empfohlene Therapiedauer wurde dabei nicht selten überschritten, vor allem im Falle von $\mathrm{Pa}$ racetamol und Ibuprofen $(62 \% ; n=115)$, seltener bei Kombinationspräparaten $(28 \% ; n=51)$ oder Triptanen, Opioiden bzw. Ergotamin (10\%). Eine prophylaktische Behandlung erhielten nur $4 \%$. Großes Vertrauen genossen auch komplementär- und alternativmedizinische Behandlungsmaßnahmen (CAM) wie Phy- siotherapie, Akupunktur und Chirotherapie.

Fazit: $46 \%$ der Patienten nahmen zu häufig schmerzstillende Mittel ein. Um die Situation zu verbessern und vor allem medikamenteninduzierte Kopfschmerzen zu vermeiden, fordern die Autoren eine engere Vernetzung von Arzt und Patient. Zudem müsse größeres Augenmerk auf prophylaktische Maßnahmen gelegt werden, um Schmerzmittelmissbrauch zu verhindern.

Dagmar Kraus

Kristoffersen ES et al. Management of primary chronic headache in the general population: the Akershus study of chronic headache. J Headache Pain. 2012;13(2):113-20. 